\title{
Performance Evaluation of IEEE STD 802.16d Transceiver
}

\author{
Mrunal P. Pathak, S. A. Shirsat \\ Electronics and Telecommunication department, Student, ME (VLSI and Embedded System), \\ Sinhgad college of engineering, University Of Pune, Maharashtra, India. \\ Electronics and Telecommunication department, Assistant Professor, \\ Sinhgad college of engineering, University Of Pune, Maharashtra, India
}

\begin{abstract}
WiMAX ("Worldwide Interoperability for Microwave Access") technology is developed to meet the growing demand of increased data rate and accessing the internet at high speeds. 802.16 family of standards is officially called Wireless MAN in IEEE. Orthogonal frequency division multiplexing (OFDM) is multicarrier modulation technique used in IEEE 802.16d (fixed WiMAX) communication standard. OFDM is used to increase data rate of wireless medium with higher spectral efficiency. The proposed work is to evaluate performance of IEEE Std 802.16d transceiver in MATLAB R2009b simulink environment. System performance evaluated using BER vs SNR for different modulation technique such as 4 QAM, 16 QAM, 64 QAM under different channel condition.
\end{abstract}

Keywords - 802.16d, MATLAB, OFDM, wimax.

\section{Introduction}

Wireless communication provide wireless connection and data transfer to connect with world anywhere and anytime. Most of application demands high data rate with more coverage area. To achieve these requirements IEEE introduced 802.16 standards. 802.16 family of standards is officially called WirelessMAN in IEEE, it has been commercialized under the name "WiMAX". It is used for wide coverage upto $40 \mathrm{Km}$ with data rates of $100 \mathrm{Mbps}$ [1]. It is used for fixed broadband wireless access.

Modulation technique used in IEEE std $802.16 \mathrm{~d}$ is OFDM (Orthogonal frequency division multiplexing). OFDM is spectrally efficient modulation technique due to orthogonality principle. It is computationally efficient due to the use of IFFT/FFT techniques to implement modulation/ demodulation functions. In an OFDM scheme, a large number of orthogonal, overlapping, narrow band sub-channels or subcarriers are transmitted in parallel. Advantages of OFDM are bandwidth efficiency, less intercarrier interference (ICI) and less intersymbol interference (ISI).

Performance parameter of OFDM baseband transceiver IEEE std 802.16d is studied using simulation. For simulation of system MATLAB R2009b is used. This OFDM system is able to support different M-QAM modulation schemes.

This paper divides in 6 sections. Section 2 explains OFDM fundamentals. System block diagram is described in section 3. Matlab based experimentation and results are presented in Section 4 and 5. Concluding remarks are given in section 6 .

\section{Literature Review}

Author [3] implements OFDM using System generator and Matlab \& Simulink. The results presented show that it is possible to implement an OFDM modulator for IEEE Std. 802.16 using Virtex II. These result shows that configurable system can be implemented for different modulation technique. Implementation of the FEC module, demodulator is future work for their project.

Author [4] describes a high level implementation of a high performance FFT for OFDM modulator and demodulator of 802.16d. The design has been coded in Verilog and targeted into Xilinx Spartan3 field programmable gate arrays. The design of the FFT is implemented and applied to fixed WiMAX-IEEE 802.16d communication standard.

Author [6] presents FPGA implementation of the Reed- Solomon decoder for use in IEEE 802.16 WiMAX systems.The decoder is based on $\operatorname{RS}(255,239)$ code, and is additionally shortened and punctured according to the WiMAX specifications. A Simulink model based on the System Generator library of low-level Xilinx blocks was used for simulation and hardware implementation.

Author [7] presents the design and implementation of OFDM system. Author has tested system performance by considering various design parameters using MATLAB 2011. All modules are designed using VHDL programming language. 


\section{Ofdm System}

OFDM is a special case of multi-carrier modulation technique, where a single data stream is transmitted over a number of lower rate sub-carriers. On classical frequency division multiplexing the total band is divided into $\mathrm{N}$ non-overlapping frequency channels, while on OFDM the band is divided into a number of overlapping frequency channels but with orthogonal frequencies. These orthogonal frequencies are obtained by using the IFFT. An OFDM symbol is formed by the sum of $\mathrm{N}$ orthogonal frequency signals, and then to avoid inter-symbol interference, a guard interval greater than the expected delay is added, during that time the signals are replicated on a cyclic prefix to prevent the effect of selective frequency delay. At baseband, an OFDM signal can be represented by a sum of modulated complex exponentials as (1).

$$
S(t)=\sum_{k=0}^{N-1} X k \exp (j 2 \pi \Delta f t)
$$

where $\mathrm{X}_{\mathrm{k}}$ is a complex number representing a BPSK, QPSK, or 16 QAM baseband symbol modulating the $\mathrm{kth}$ subcarrier and $\Delta \mathrm{f}$ is the subcarrier spacing. OFDM system is as shown in Fig 1.

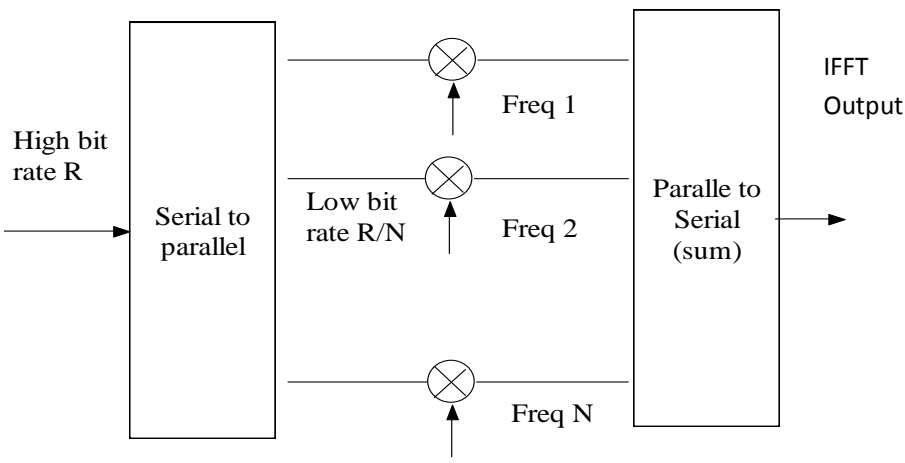

Fig 1 Multicarrier FDM or OFDM [8]

III. System block diagram

Physical layer of IEEE 802.16d transmitter and receiver diagram is as shown in Fig. 2.

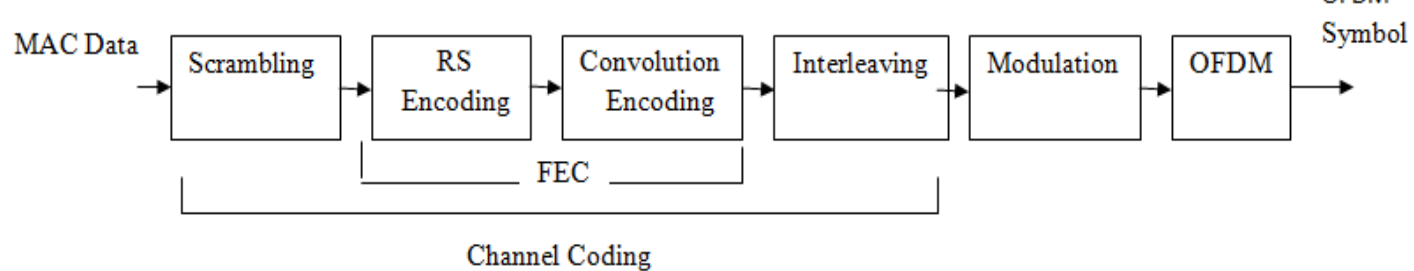

(a)

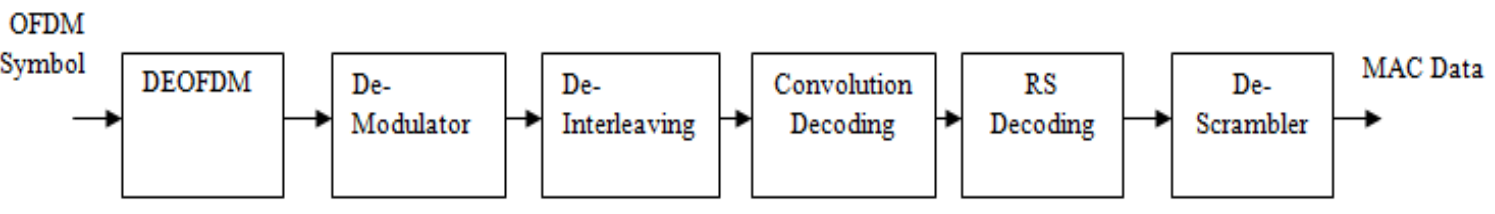

(b)

Fig 2 a 802.16d transmitter block diagram for the OFDM PHY [1]

b 802.16d receiver block diagram for the OFDM PHY [1]

\subsection{Transmitter}

The main components of OFDM transmitter are shown in Fig. 2a. The scrambler also known as randomizer is used as random bit generator. The first three blocks are used for data coding and interleaving. The coded bits will be mapped by the constellation modulator. The OFDM consist of serial to parallel converter which converts the data bits from the serial form to the parallel form. The Inverse Fast Fourier Transform (IFFT) transforms the signals from the frequency domain to the time domain, an IFFT converts a number of complex data points, of length that is power of 2 , into the same number of points but in the time domain. The number of subcarriers determines how many sub-bands the available spectrum is split into. The Cyclic Prefix (CP) is a copy of the last $\mathrm{N}$ samples from the IFFT, which are placed at the beginning of the OFDM frame to overcome ISI problem. It is important to choose the minimum necessary CP to maximize the bandwidth efficiency of the system. 


\subsection{Receiver}

The main blocks of OFDM receiver are as shown in Fig. 2b. The received signal goes through the cyclic prefix removal and a serial-to-parallel converter. After that, the signals are passed through an $\mathrm{N}$-point fast Fourier transform to convert the signal to frequency domain. The output of the FFT is formed from the first M samples of the output. The reverse of forward error correction block obtained by convolutional decoder, RS decoder and de-scrambler. The recovered signal get same as transmitted by transmit signal.

\subsection{Modulation}

OFDM physical layer is adaptive and supports different modulation techniques. Table 1 summarized channel coding parameters for various modulation techniques.

TABLE 1

Channel coding per modulation technique for $802.16 \mathrm{~d}$ [1]

\begin{tabular}{|l|l|l|l|l|c|}
\hline Modulation & $\begin{array}{l}\text { Uncoded } \\
\text { block size } \\
\text { (bytes) }\end{array}$ & $\begin{array}{l}\text { Coded } \\
\text { block } \\
\text { size } \\
\text { (bytes) }\end{array}$ & $\begin{array}{c}\text { Overal } \\
\text { l rate }\end{array}$ & RS- code & $\begin{array}{c}\text { CC code } \\
\text { Rate }\end{array}$ \\
\hline BPSK & 12 & 24 & $1 / 2$ & $(12,12,0)$ & $1 / 2$ \\
\hline 4 QAM & 24 & 48 & $1 / 2$ & $(32,24,4)$ & $2 / 3$ \\
\hline 4 QAM & 36 & 48 & $3 / 4$ & $(40,36,2)$ & $5 / 6$ \\
\hline 16-QAM & 48 & 96 & $1 / 2$ & $(64,48,8)$ & $2 / 3$ \\
\hline 16-QAM & 72 & 96 & $3 / 4$ & $(80,72,4)$ & $5 / 6$ \\
\hline 64-QAM & 96 & 144 & $2 / 3$ & $(108,96,6)$ & $3 / 4$ \\
\hline 64-QAM & 108 & 144 & $3 / 4$ & $(120,108,6)$ & $5 / 6$ \\
\hline
\end{tabular}

\subsection{Physical Layer Specification for OFDM of 802.16d.}

Table 2 shows the physical layer specifications of $802.16 \mathrm{~d}$ for OFDM module.

TABLE 2

Physical layer Specification [1]

\begin{tabular}{|l|l|l|}
\hline IFFT length & $\mathrm{Nfft}$ & 256 \\
\hline Bandwidth & $\mathrm{B}$ & $10 \mathrm{MHz}$ \\
\hline No of subcarrier & $\mathrm{Nc}$ & 256 \\
\hline Subcarrier spacing & $\mathrm{F}=\mathrm{B} / \mathrm{Nc}$ & $39.06 \mathrm{KHz}$ \\
\hline Useful symbol time & $\mathrm{Tfft} / \mathrm{Tb}=1 / \mathrm{F}$ & $25.6 \mathrm{usec}$ \\
\hline Sampling time & $\mathrm{Ts}=\mathrm{Tofdm} / \mathrm{Nfft}$ & $0.1 \mathrm{usec}$ \\
\hline Guard interval & $64 * \mathrm{Ts}$ & $6.4 \mathrm{usec}$ \\
\hline Signal duration & $\mathrm{Tsignal}=\mathrm{Tb}+\mathrm{Tg}$ & $32 \mathrm{usec}$ \\
\hline
\end{tabular}

IV. Transceiver Model Of IEEE STD 802.16d

Fig 3 shows transceiver model of IEEE STD 802.16d simulated using MATLAB and SIMULINK.

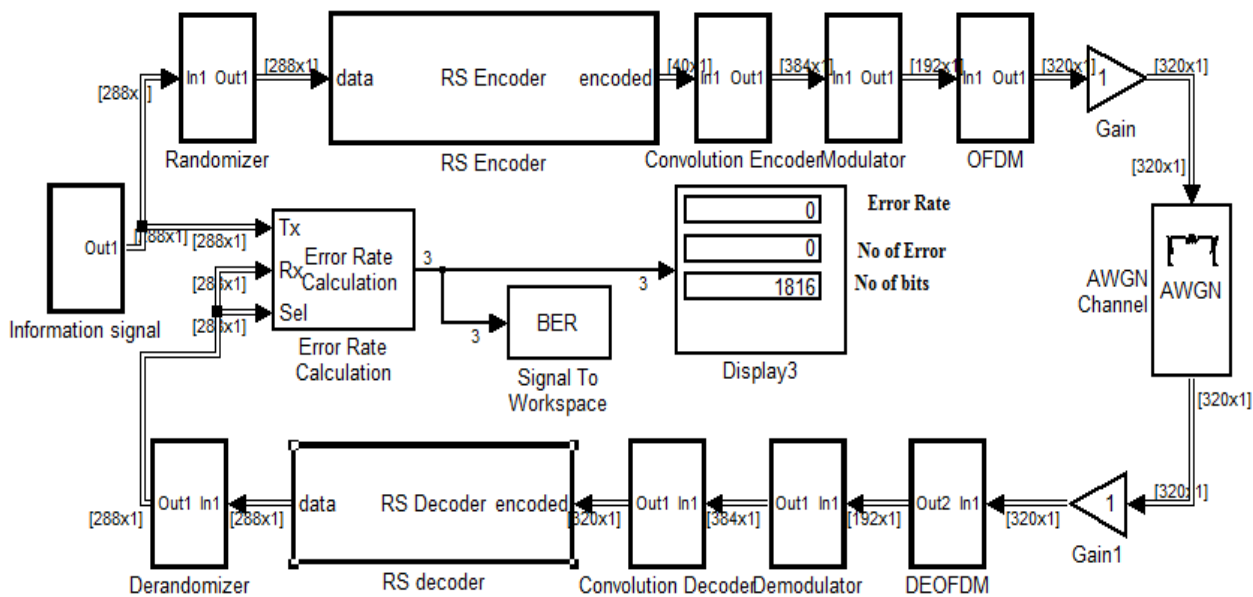

Fig 3 Simulink diagram of 802.16d OFDM PHY layer for 4 QAM modulation.

The simulation parameters used are: 
Number of subcarriers: 256 (for FFT)

Modulation Scheme: 4 QAM, 16 QAM and 64 QAM

Channel: AWGN

As shown in Fig. 3 Bernoulli binary this data source acts as information signal is fed to the baseband transmitter. As specified in the standard, 802.16d OFDM PHY layer baseband transmitter is composed of three major parts: channel coding, modulation, and OFDM transmitter. For the receiver complementary operations are applied in the reverse order. The structure of IEEE 802.16d OFDM PHY layer is shown in Fig. 3. The first block in the transmitter is randomizer. The timing recovery problem at the receiver is minimized by use of randomizer which prevent a long sequence of $1 \mathrm{~s}$ and $0 \mathrm{~s}$. In $802.16 \mathrm{~d}$ standard the randomizer is implemented with a 15 bits shift register and XOR gates. Error detection and correction is carried out by forward error correction block which is composed of Reed-Solomon encoder, convolutional encoder and puncture (used to adjust different data rate). These are the mandatory blocks on the standard FEC, consisting of the concatenation of a Reed-solomon outer code and a rate-compatible convolutional inner code. The modulation used in $802.16 \mathrm{~d}$ is integer-mapped 4 QAM, 16 QAM, 64 QAM.

OFDM transmitter is composed of three parts: assemble OFDM frame, create OFDM signal by performing IFFT/FFT, and cyclic prefix. Data is sent in the form of OFDM symbols. Accurate synchronization is necessary to ensure correct baseband processing. OFDM systems are very sensitive to frequency offset because there might be loss of orthogonality between sub symbols. Accurate synchronization is necessary to ensure correct baseband processing. OFDM systems are very sensitive to frequency offset because there might be loss orthogonality between sub symbol.

\section{Simulation Results}

Systematic computer simulations were carried out to evaluate the Bit Error Rate (BER) performance of IEEE 802.16d.Bit error rate is the rate at which errors occur in a transmission system. In mathematical form it idefined as:BER = number of errors / total number of bits sent.The calculation gives, how system performs?The platformemployed for performing the simulations is MATLAB vR2009b.

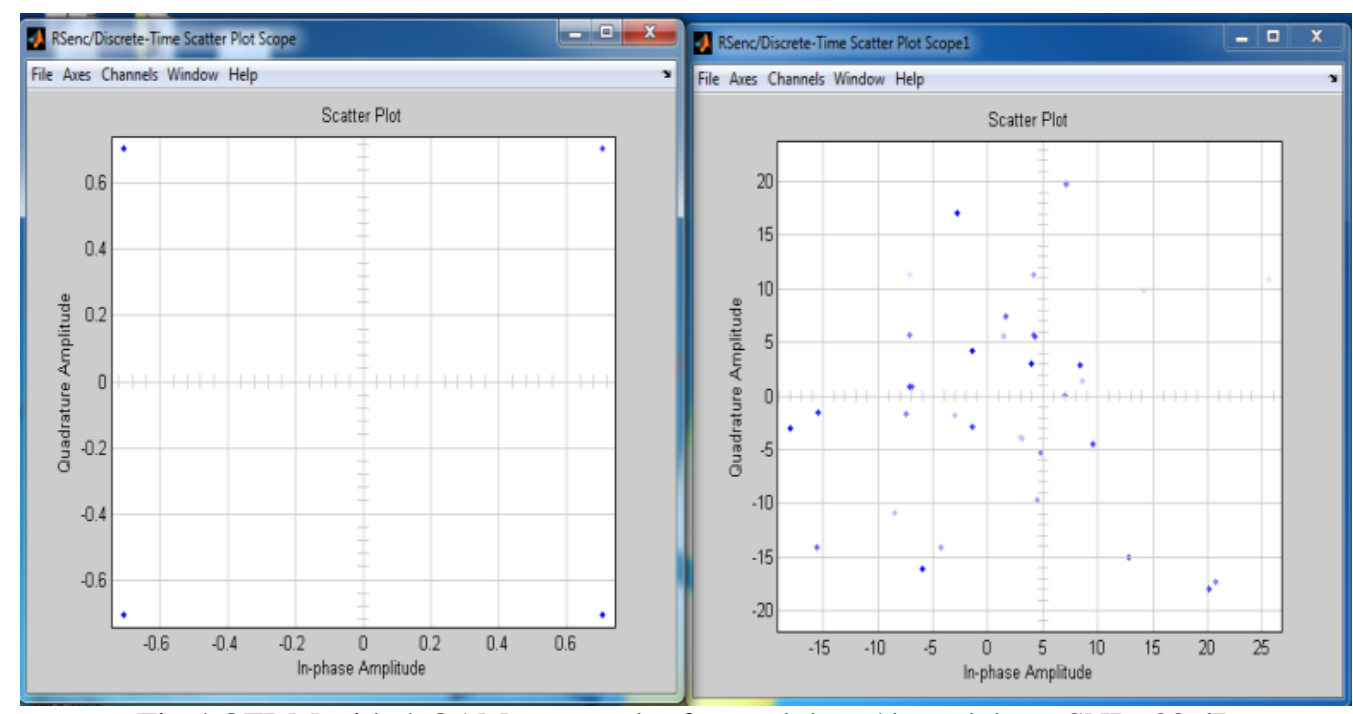

Fig 4 OFDM with 4-QAM scatter plot for modulator/demodulator SNR=20 dB

Fig. 4 shows the scatter plot of transmitted and received data. Received scatter plot consist of noise in signal of AWGN at SNR= 20dB. Fig. 5 shows 15 bit transmitted and received data which shows receiver get transmitted data. 


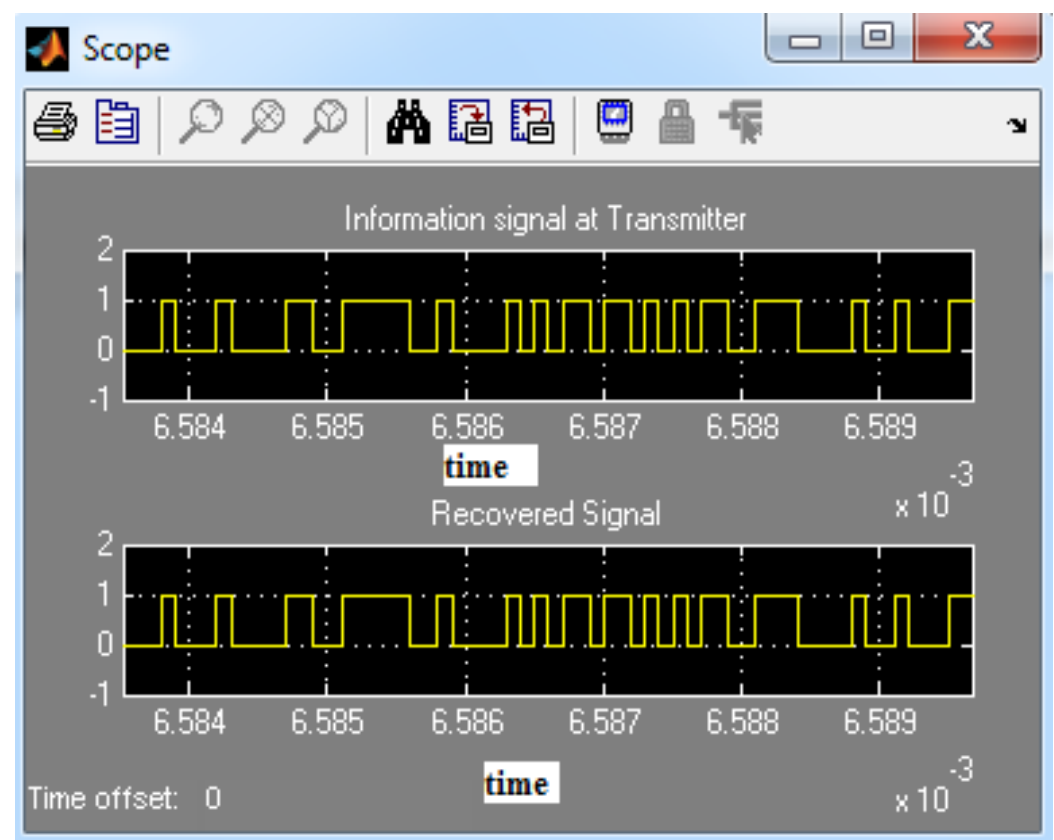

Fig 5 Transmitted and recovered signal.

Fig. 6 show a comparison of the simulation results for 4-QAM, 16-QAM and 64-QAM for AWGN channel. Simulation results shows that 4 QAM has good BER vs Eb/No performance than 16 QAM and 64 QAM. 64 QAM is spectrally efficient as it transfer $8 \mathrm{bps} / \mathrm{Hz}$ where as 4 QAM $2 \mathrm{bps} / \mathrm{Hz}$. This shows there is trade off between spectral efficiency and BER vs Eb/No.

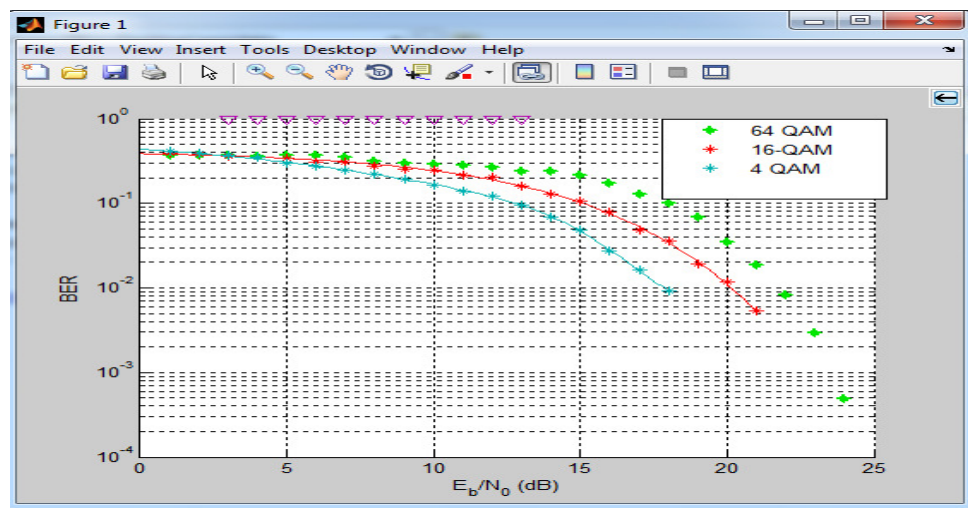

Fig 6 BER for received signal for AWGN channel

Fig. 7 shows the performance of system for different modulation techniques with Rayleigh fading channel effect. 4 QAM has less BER rate for higher Eb/No. 4 QAM is better BER rate than 16 QAM and 64 QAM for higher value of $\mathrm{Eb} / \mathrm{No}$.

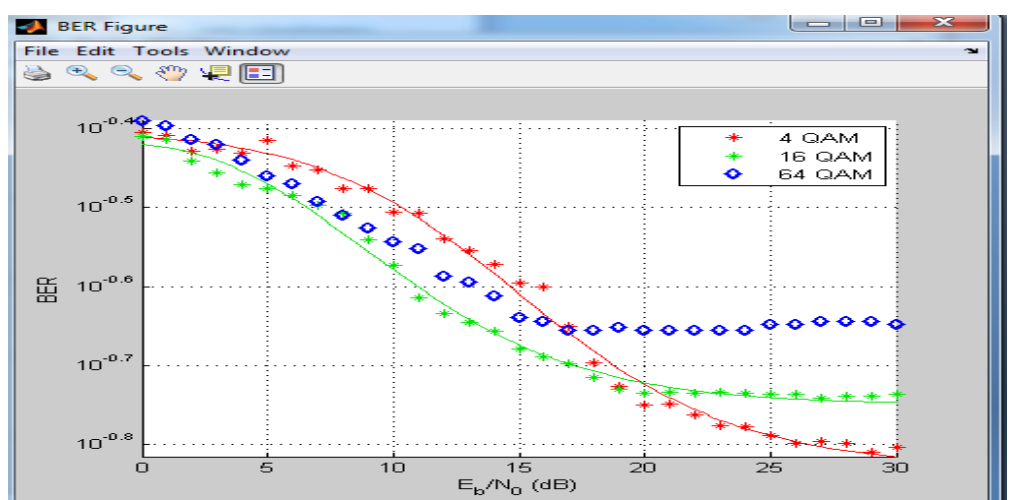

Fig 7 BER for received signal for Rayleigh channel 
Fig. 8 shows comparison between AWGN and Rayleigh channel. The performance for AWGN channel is best than Rayleigh channel as it has the lower BER under 4 QAM. It is same for 16-QAM and 64-QAM modulation techniques.

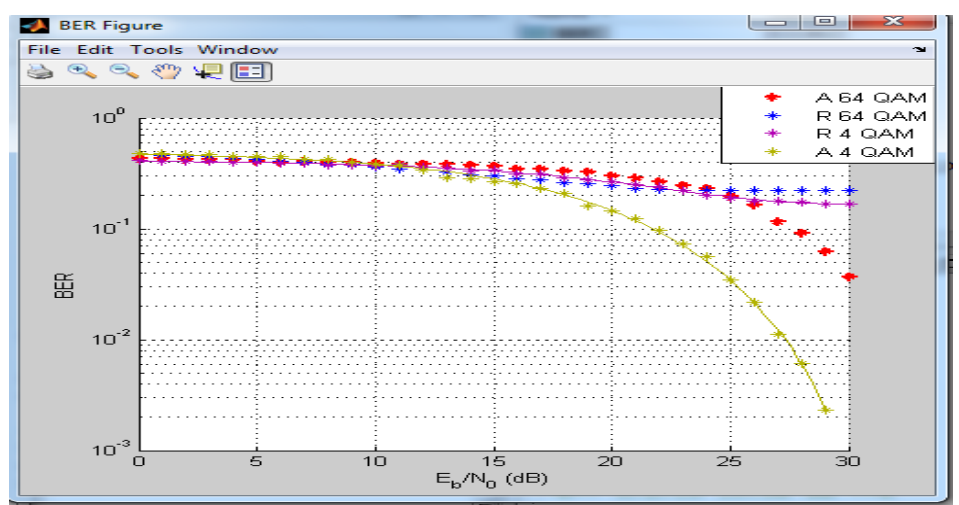

Fig 8 BER performance of AWGN and Raleigh Channels for 4-QAM and 64-QAM modulation techniques.

\section{Conclusion}

The performance of an OFDM based WiMAX communication system with different digital modulation techniques and channel is studied. Receiver gets same data as transmitted from transmitter. 4 QAM modulation techniques has good BER vs Eb/No performance for both AWGN and Rayleigh channel.

BER performance for AWGN channel is better than Rayleigh channel. WiMax Transceiver simulated in MATLAB shows better performance for AWGN channel than fading channel (Rayleigh channel). The next objective of this work is to design and implement baseband OFDM transceiver on FPGA hardware.

\section{Acknowledgement}

I am glad to express my sincere thanks to Prof. S. A. Shirsat who offered me valuable and constructive tips for my work.

\section{References}

[1] Abbas S., Khan W.A., Khan T.A., Ahmed S. "Implementation of OFDM Baseband Transmitter Compliant IEEE Std 802.16d on FPGA" Publication Year: 2010 , Page(s): 22 - 26.

[2] M.A. Mohamed, A.S. Samarah, M.I. Fath Allah "Study of performance parameters effects on OFDM systems" IJCSI International Journal of Computer Science Issues, Vol. 9, Issue 3, No 2, May 2012.

[3] Joaquin Garcia, Rene Cumplido Department of Computer Science, "On the design of an FPGA-Based OFDM modulator for IEEE 802.16-2004” INAOE, Puebla, Mexico 2005 International Conference on Reconfigurable Computing and FPGAs (ReConFig 2005).

[4] K. Harikrishna, T. Rama Rao, and Vladimir A. Labay, "FPGA Implementation of FFT Algorithm for OFDM Based IEEE 802.16d (Fixed WiMAX) Communications" Journal Of Electronic Science And Technology, Vol. 8, No. 3, September 2010

[5] IEEE computer society and the IEEE microwave theory \& techniques society " IEEE standard for Local and Metropolitan area network. Part 16: Air interface for fixed broadband wireless access system." 1 October 2004.

[6] Miljko Bobrek, Kenyon H. Clark, Austin P. Albright "FPGA Implementation of Reed-Solomon Decoder for IEEE 802.16 WiMAX Systems using Simulink-Sysgen Design Environment” Oak Ridge National Laboratory Cognitive Radio Program 1 Bethel Valley Rd Oak Ridge ,TN 37831.

[7] M.A. Mohamed, A.S. Samarah, M.I. Fath Allah, "Implementation of the OFDM Physical Layer Using FPGA" IJCSI International Journal of Computer Science Issues, Vol. 9, Issue 2, No 2, March 2012.

[8] Bernard Sklar, Prabitra Kumar Ray, "Digital Communication (Fundamental and applications)".

[9] Andreas F. Molisch, "Wireless Communication" 